\title{
Peripherally administered KP-10 prevents development of insulin-induced hypoglycemic shock in diabetic rhesus monkeys
}

\author{
IZ Qureshi, I Fatima \\ Laboratory of Animal and Human Physiology, Department of Animal Sciences, Quaid-i-Azam University, \\ Islamabad, Pakistan
}

Received: August 15, 2019

Accepted: September 22, 2019

\begin{abstract}
Aim: This study investigated whether kisspeptin-10 (KP-10) prevents diabetic rhesus monkeys from insulin-induced hypoglycemic shock. Materials and methods: Thirty-six adult male rhesus monkeys were used, six in each group. Diabetes was induced with streptozotocin ( $45 \mathrm{mg} / \mathrm{kg}$ b.w.; single dose i.v.). Groups were as: saline control, insulin alone, pre-insulin (treated with KP-10, 30 min before insulin), post-insulin (treated with KP-10, 30 min after insulin), treated with premix dose of KP-10 $(50 \mu \mathrm{g})$ and insulin, and the group treated with the kisspeptin antagonist P234 $(50 \mu \mathrm{g})$. Following an overnight fast, each animal was subjected to respective treatment, and blood glucose concentrations were recorded every 30-min interval for $3 \mathrm{~h}$. Results: Intergroup comparisons demonstrated that treatment with KP-10 prior to insulin administration and kisspeptin-insulin premix treatment allowed blood glucose levels to rise to significantly higher levels $(p<0.001)$ by $180 \mathrm{~min}$ in diabetic and healthy animals compared to treatment with insulin alone. However, intragroup comparisons revealed a significant decrease in blood glucose level in diabetic animals only. Treatment with P234 antagonist followed by insulin administration abolished the preventive action of kisspeptin, whereby blood glucose decreased significantly $(p<0.001)$ in both diabetic and healthy animals. KP-10 post-insulin treatment, however, remained ineffective and led, instead, to significantly decreased glucose concentrations by $180 \mathrm{~min}$ in both diabetic and healthy animals when compared to animals treated with insulin alone. Conclusions: KP-10 bears therapeutic potential to prevent hypoglycemic shock that may sometimes occur during intensive insulin therapy. Several pharmacological aspects of its interaction with insulin and other drugs, however, remain to be investigated.
\end{abstract}

Keywords: hypoglycemic shock, diabetes, kisspeptin, insulin-induced hypoglycemia, rhesus monkeys

\section{Introduction}

Defective glucose homeostasis caused by $\beta$-cell loss and subsequent insulin dysfunction in diabetes mellitus leads to the manifestation of secondary complications (22). Hypoglycemia is one of the most common complications in diabetic patients that may prove fatal under certain circumstances (16). In clinical practice, hypoglycemia is usually prevalent in those patients who are undergoing treatment with insulin or insulin secretagogues (4). This is mainly due to the reason that normal physiological responses of the body are compromised in both type I and type II diabetes, ultimately developing a progression of hypoglycemic state (21). Various animal models have been used to better understand diabetes pathogenesis and to devise treatment strategies. Non-human primates are considered the most suitable models because of the

Corresponding author: Irfan Zia Qureshi, $\mathrm{PhD}$

Laboratory of Animal and Human Physiology, Department of Animal Sciences, Quaid-i-Azam University, P.O. Box 45320, Islamabad, Pakistan

Phone: +92 5190643 013; Mobile: +92 3365255 068; Fax: +92 512 601176; E-mail: irfanzia@qau.edu.pk 
common occurrence of diabetes and pathologic changes in the endocrine pancreas similar to those encountered in humans. In addition, they are suitable test subjects for newly discovered pharmacological agents (9). Studies over the past decade have revealed the regulatory role of kisspeptin in glucose homeostasis. A recent study in mice showed that KP-10 plays a direct role in modulating the energy balance in the adipose tissue, which was reciprocally linked to testicular activities (2). This development has opened a new portal to further investigations in this direction (13). The presence of KISS1/GPR54 in the endocrine pancreas implicates an intraislet function for regulation of insulin secretion in response to fluctuating glucose levels (10).

In addition to insulin and glucagon, communication between different organs (12) has further highlighted the role of kisspeptin as a glucoregulatory hormone. Glucagon induces gluconeogenesis in liver tissue and promotes hepatic Kiss-1 expression. Increased circulating kisspeptin levels ultimately downregulate insulin secretion from the $\beta$ cells (20). In vitro experiments on mouse pancreatic islets have revealed that, in the presence of stimulatory glucose levels, kisspeptin promotes insulin secretion (3). Similarly to other peptide-based drugs (14), the glucoregulatory role of kisspeptin indicates its therapeutic potential in treating diabetes. Pharmacological studies on the regulatory role of kisspeptin following exogenous insulin overload are, however, still awaited. In this respect, the aim of this study was to test the therapeutic potential of kisspeptin. As a first step, we asked the question whether kisspeptin infusion helps in preventing the hypoglycemic effect of exogenous insulin treatment in healthy and diabetic states. To test this hypothesis, diabetic and healthy rhesus monkeys were subjected to different sets of insulin and kisspeptin-10 (KP-10) treatments. Determination of circulating blood glucose levels was the end point in each set of experiments.

\section{Materials and Methods}

Ethical approval for this study was obtained from the "Bio-Ethical Committee" of Quaidi-Azam University on use of Animals for Scientific Research (BBC-FBS-QAU/2017-31B). Thirty-six healthy adult male rhesus monkeys (Macaca mulatta; 5-6 years old with an average body weight of $9.98 \pm 1.33 \mathrm{~kg}$ ) were obtained from the Federal Wildlife Department, Islamabad, Pakistan. Depending on the plasma clearance of drugs, the monkeys could be reused for further experiments to minimize the number of experimental animals, but to avoid bias and erroneous results that might occur due to pharmacological intervention, separate monkeys were used for all groups.

\section{Maintenance and care}

Monkeys were housed in the Primate Facility of the Department of Animal Sciences, Quaid-iAzam University, Islamabad, in appropriately designed cages. They were maintained on a diet of bread, fresh fruits, and vegetables supplemented with eggs and nuts. All animals had free access to drinking water. To minimize the stress that might occur due to handling and venous catheterization, monkeys were gradually habituated to chair restraint for 6 weeks with daily training sessions, each session of continuous $3 \mathrm{~h}$. The behavior of each animal was monitored regularly during the restraining period for untoward signs or symptoms especially in diabetic monkeys.

\section{Drugs and chemicals}

Heparin (Kota Bharu, Kelantan, Malaysia), insulin (Humulin ${ }^{\circledR}$ R; Eli Lilly and Company, Egypt), and Ketamax (Rotex, Medica, Trittau, Germany) were purchased locally. 
Streptozotocin (STZ) was obtained from Gene Linx International Inc. (USA). KP-10 or metastin 45-54 (KiSS-1; 112-121; H-YNWNSFGLRF-NH 2 ) was obtained as lyophilized powder from Calbiochem (La Jolla, CA, USA). Kisspeptin antagonist P234 was purchased from Sigma-Aldrich Chemical Co. (St. Louis, MO, USA).

\section{Preparation of diabetic model}

After proper restraining and habituation as mentioned above, monkeys were successfully made diabetic with a single intravenous (i.v.) injection of STZ at the dose of $45 \mathrm{mg} / \mathrm{kg}$ b.w. This was accomplished approximately 2 weeks earlier than the start of actual experiments. This was to ensure that the desired hyperglycemic levels were maintained. Diabetic symptoms appeared within 3-5 days of treatment. Fasting and post-prandial glucose levels were monitored regularly to confirm the overt diabetes. Insulin (Humulin ${ }^{\circledR} \mathrm{R}$ ) treatment was provided where circulating glucose appeared approaching dangerously high levels. Following $12 \mathrm{~h}$ of fast, animals having fasting blood glucose levels in the range of $180-220 \mathrm{mg} / \mathrm{dl}$ were included in the study, whereas those who developed insulin resistance were excluded from the experiments.

\section{Venous catheterization}

For sequential blood sampling, overnight fasted (20:00-08:00 h) monkeys were catheterized with sterile $0.8 \mathrm{~mm} / 22 \mathrm{G}$ Teflon cannulas inserted into the saphenous vein. Briefly, each animal was sedated with light ketamine $\mathrm{HCl}(10 \mathrm{mg} / \mathrm{kg}$ b.w.; i.m.). The saphenous vein was exposed after shaving and scrubbing with $70 \%$ ethanol. A cannula was then inserted into the vein and secured with adhesive tape. The animal was then subjected to chair restraint for experimental dose administration.

\section{Insulin challenge}

As a standard procedure, all animals were subjected to overnight fast. The following morning, after gaining full recovery from ketamax sedation, monkeys were intravenously administered insulin doses in accordance with the blood glucose level of each animal. Very similarly to humans, insulin doses were optimized for both healthy and diabetic monkeys according to variations of the physiological responses of each animal. Approximately, $6.5-9.0$ and 7-11 IU/kg b.w. of insulin doses were found sufficient for the reduction of blood glucose levels in healthy and diabetic monkeys, respectively. The physical condition of the animals was monitored for general restlessness and untoward signs relating to severe hypoglycemia.

\section{Experimental design}

Animals were fasted overnight, and actual treatment was started at 08:00 h. Administration of sedatives, i.v. catheterization for drug administration and blood sampling, and chairrestraint procedure took approximately $30 \mathrm{~min}$. Sedation and insertion of cannulas were completed within $20 \mathrm{~min}$, whereas animals were chair-restrained within 5-10 min by the expert handlers. Later, animals were allowed to relax for an additional $15 \mathrm{~min}$. The reason for this was that the whole procedure was started at 07:00 h to get the animals ready for the treatment. Predose glucose readings were recorded first. After administration of treatment doses, glucose readings were taken sequentially for $3 \mathrm{~h}$ (180 min). The time interval between samples was $30 \mathrm{~min}$. Monkeys were monitored carefully for any symptoms of severe hypoglycemia. Readings were taken at $-60,-30,0,30,60,90,120,150$, and $180 \mathrm{~min}$ from control and treated animals. Only $100-200 \mu \mathrm{l}$ of blood was withdrawn to 
monitor changes in the circulating blood glucose level. Thus, there was no chance of hypovolemia or anemia.

In all groups, the saline, KP-10, and insulin doses were administered i.v. Healthy and diabetic monkeys underwent the following treatments:

1. Vehicle: saline treatment $(0.90 \% ; 1 \mathrm{ml})$.

2. Insulin: insulin alone treatment (according to b.w.)

3. Pre-insulin KP: bolus dose of KP-10 $(50 \mu \mathrm{g})$ as described by Wahab et al. (24) was administered $30 \mathrm{~min}$ before the insulin treatment.

4. Kisspeptin antagonist P234 administration: P234 (38 $\mu \mathrm{g} \cdot \mathrm{kg}^{-1} \cdot \mathrm{b}$.w.) [as described by Huma et al. (11)] was given 15 min before KP-10 treatment followed by insulin administration.

5. Post-insulin KP: KP-10 was administered 30 min after insulin treatment.

6. Combined premix: calculated doses of insulin (according to the b.w. of each animal) and KP-10 were administered in combination.

Food was withdrawn during the experimental period, whereas animals had free access to drinking water.

The experimental design is illustrated by the schematic diagram as shown in Fig. 1.

\section{Determination of blood glucose}

Whole blood glucose concentrations were determined with a handheld glucometer (AccuChek Active glucometer, Roche, Germany) for instant or on the spot evaluation of the glucoregulatory response of hypoglycemic animals.

\section{Statistical analyses}

Mean blood glucose concentrations within treatment groups at different intervals were compared with one-way analysis of variance using the IBM Statistical Product and Service Solutions statistical software (SPSS version 20.0, Chicago, IL, USA) followed by Tukey's

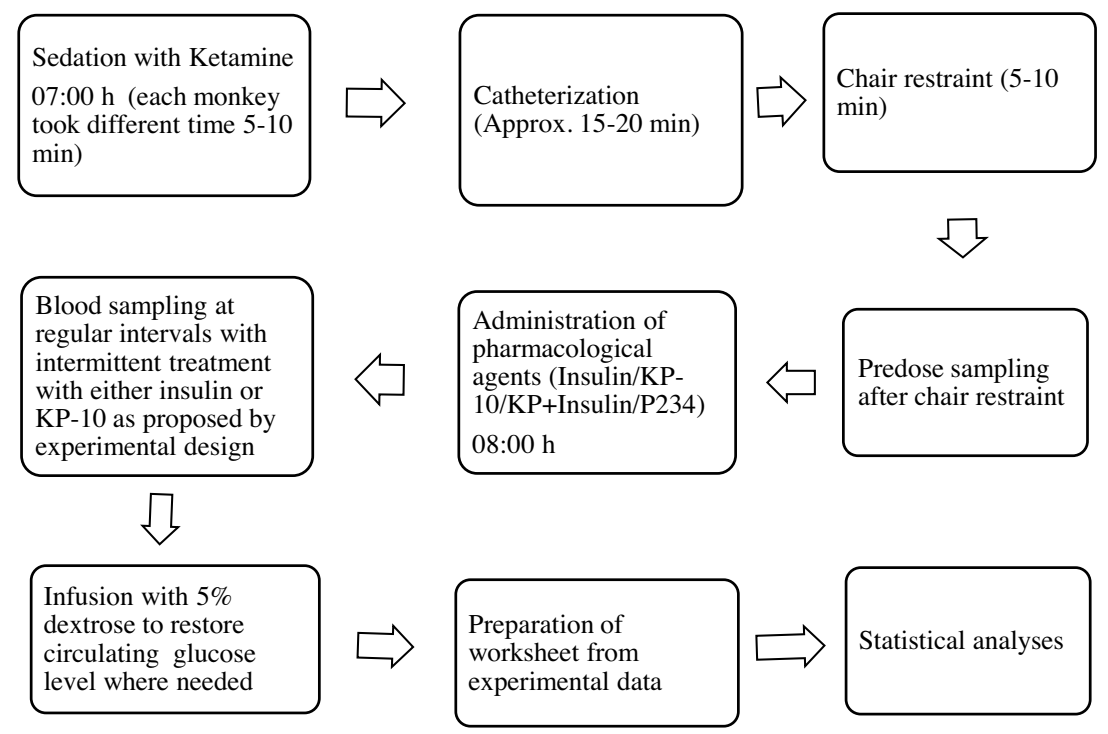

Fig. 1. A schematic diagram of experimental design 
post-hoc analysis. Comparison between insulin alone and KP-10 treated groups was made at 180 min by applying paired $t$-test using the Sigma plot (version 12.0, CA, USA). Significance level was $p<0.005$.

\section{Results}

Pharmacological treatments of diabetic monkeys

Intragroup comparisons of blood glucose levels at different time intervals in saline-treated diabetic monkeys revealed no significant change over experimental time duration as represented by pre- ( 0 -min reading) and post-treatment (180-min reading) glucose concentrations, i.e., 210 and $203 \mathrm{mg} / \mathrm{dl}$, respectively (Fig. 2a).
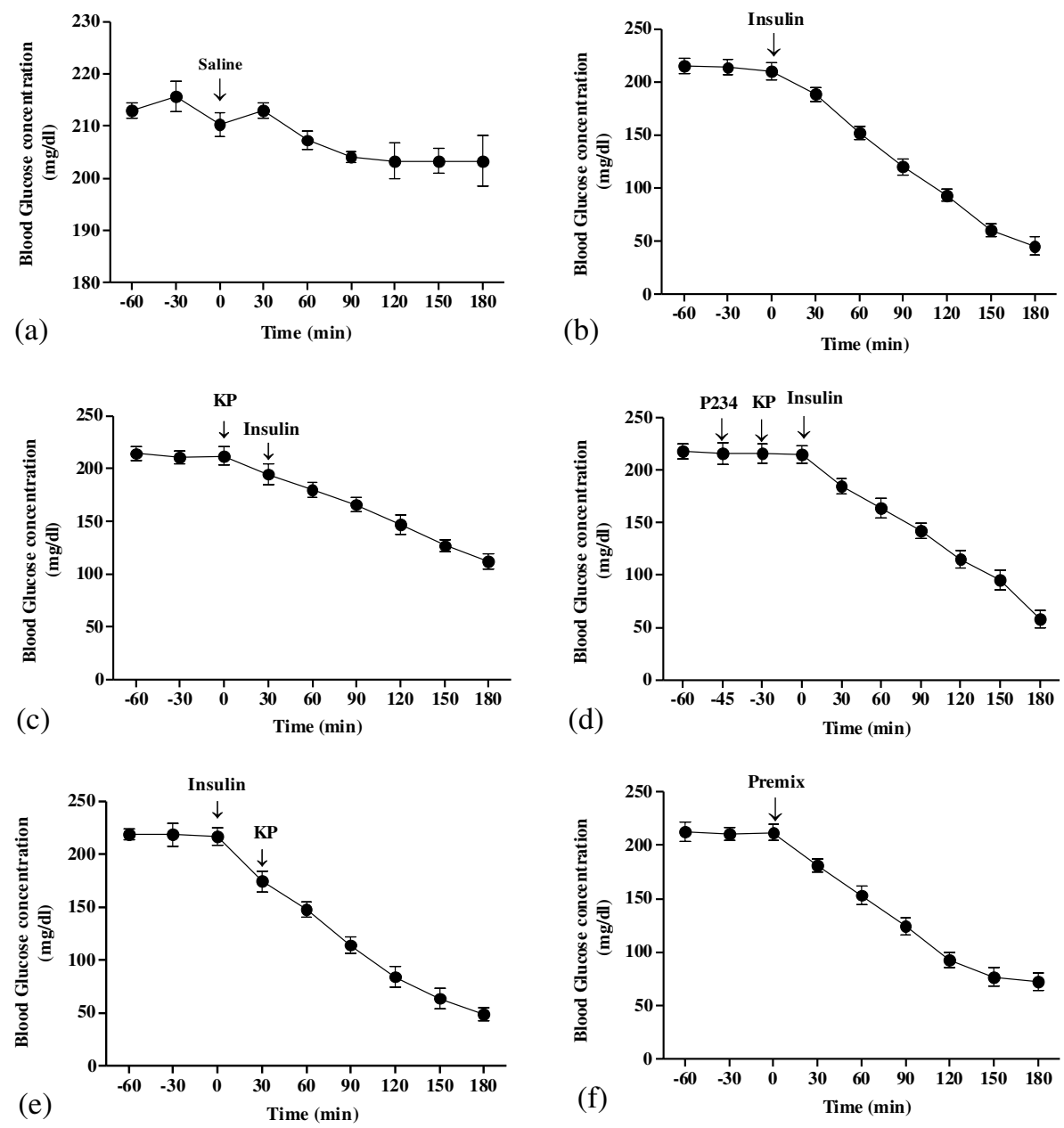

Fig. 2. Blood glucose levels $(\mathrm{mg} / \mathrm{dl})$ in diabetic male rhesus monkeys at different time points for continuous $3 \mathrm{~h}$ (180 min): (a) saline treatment, (b) treatment with insulin alone, (c) KP-10 administration prior to insulin treatment,

(d) administration of KP-10 antagonist P234 prior to KP-10 treatment, (e) KP-10 administration post-insulin treatment, and (f) treatment with a combined premix dose of KP-10 and insulin. Values are mean $\pm \mathrm{SE}$ 
In contrast, upon insulin administration, glucose levels were $210 \mathrm{mg} / \mathrm{dl}$ at $0 \mathrm{~min}$, which declined radically after $30 \mathrm{~min}$ and reached $45 \mathrm{mg} / \mathrm{dl}$ by $180 \mathrm{~min}(78.7 \%$ decline $)$. Thus, after post-insulin treatment, glucose concentrations at $180 \mathrm{~min}$ in diabetic animals showed a significant difference $(p<0.001)$ compared to predose glucose concentrations (Fig. 2b). Treatment with KP-10 half an hour before insulin administration brought about a non-significant decrease in circulating glucose levels. At $-30 \mathrm{~min}$, glucose levels were $211 \mathrm{mg} / \mathrm{dl}$, soon after which KP-10 was infused. Blood glucose level was $212 \mathrm{mg} / \mathrm{dl}$ at 0 -min post-KP treatment, reflecting no alteration. Administration of insulin at 0 -min post-KP treatment led to a start of decline in blood glucose by $30 \mathrm{~min}(195 \mathrm{mg} / \mathrm{dl}$, an $8 \%$ decline), which further declined highly significantly $(p<0.001)$ to $112 \mathrm{mg} / \mathrm{dl}$ by 180 min (47\% decline; Fig. 2c).

In diabetic monkeys, after blocking kisspeptin action with P234, no significant change was noticeable in the glucose concentration after KP-10 treatment until the 0 -min time point ( 216 vs. $214 \mathrm{mg} / \mathrm{dl}$ ). However, soon after treatment with insulin, glucose concentration started declining rapidly and significantly $(p<0.001)$ until it reached $58 \mathrm{mg} / \mathrm{dl}$ by $180 \mathrm{~min}$. This represented a $73 \%$ decline (Fig. 2d).

When diabetic monkeys were treated first with insulin and half an hour later injected with KP-10, glucose levels decreased significantly $(p<0.001)$ over the 3 -h time period. At $0 \mathrm{~min}$, glucose levels were $217 \mathrm{mg} / \mathrm{dl}$, which decreased to $174 \mathrm{mg} / \mathrm{dl}$ (20\% decline) by $30 \mathrm{~min}$, after which KP-10 was infused. Glucose concentration then continued to decrease until it reached $48 \mathrm{mg} / \mathrm{dl}$ by $180 \mathrm{~min}$, i.e., a $78 \%$ decline compared to the 0 -min level and a $72 \%$ decline compared to the 30-min time point when KP-10 was administered (Fig. 2e).

Treatment with a premix dose of KP-10 resulted in significant decreases in glucose levels by $180 \mathrm{~min}$. At $0 \mathrm{~min}$, glucose levels were $212 \mathrm{mg} / \mathrm{dl}$, which gradually declined to $72 \mathrm{mg} / \mathrm{dl}$ by $180 \mathrm{~min}$, showing an overall 66\% significant decline $(p<0.001)$ over a 3 -h time period (Fig. 2f).

\section{Intergroup comparison of diabetic monkeys at $180 \mathrm{~min}$}

By 180 min, significantly higher $(p<0.001)$ blood glucose levels were noticeable in animals treated with KP-10 administered 30 min before insulin treatment than in the group treated with insulin alone. Animals treated with a premix dose of KP-10 and insulin also showed significantly greater glucose concentrations $(p<0.01)$ than did the group treated with insulin alone. However, no significant difference was observed between the blood glucose levels of

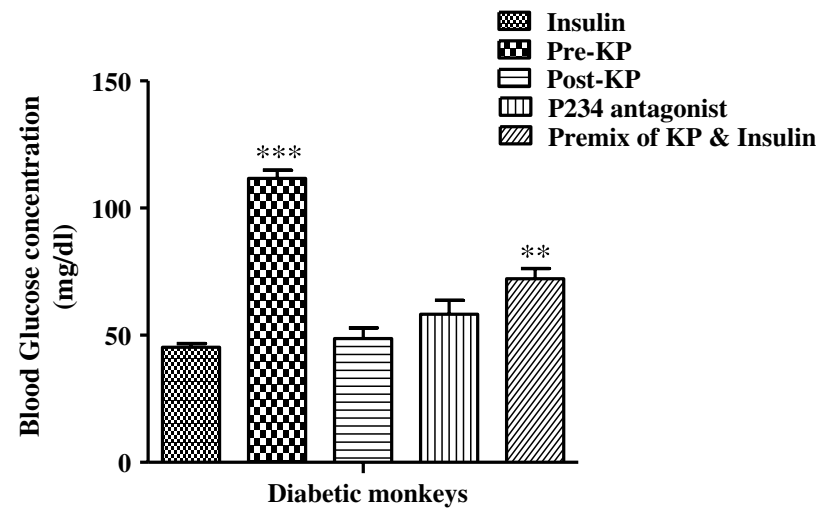

Fig. 3. Intergroup comparison of mean blood glucose in diabetic monkeys. Blood glucose levels $(\mathrm{mg} / \mathrm{dl})$ of experimental groups and the group treated with insulin alone at $180 \mathrm{~min}$. Values are mean $\pm \mathrm{SE}$ $(* * p<0.01 . * * * p<0.001)$ 
animals treated with KP-10 after 30 min of insulin administration and animals treated with insulin alone. Similarly, the P234-treated group also did not show any difference in mean blood glucose when compared with treatment with insulin alone (Fig. 3).

Pharmacological treatments of healthy non-diabetic monkeys

Intragroup comparison at different time points revealed no significant change in glucose concentration in saline-treated healthy monkeys over 3-h duration, which was demonstrated by pre- (0-min reading) and post- treatment (180-min reading) values of 85 and $98 \mathrm{mg} / \mathrm{dl}$, respectively (Fig. 4a).
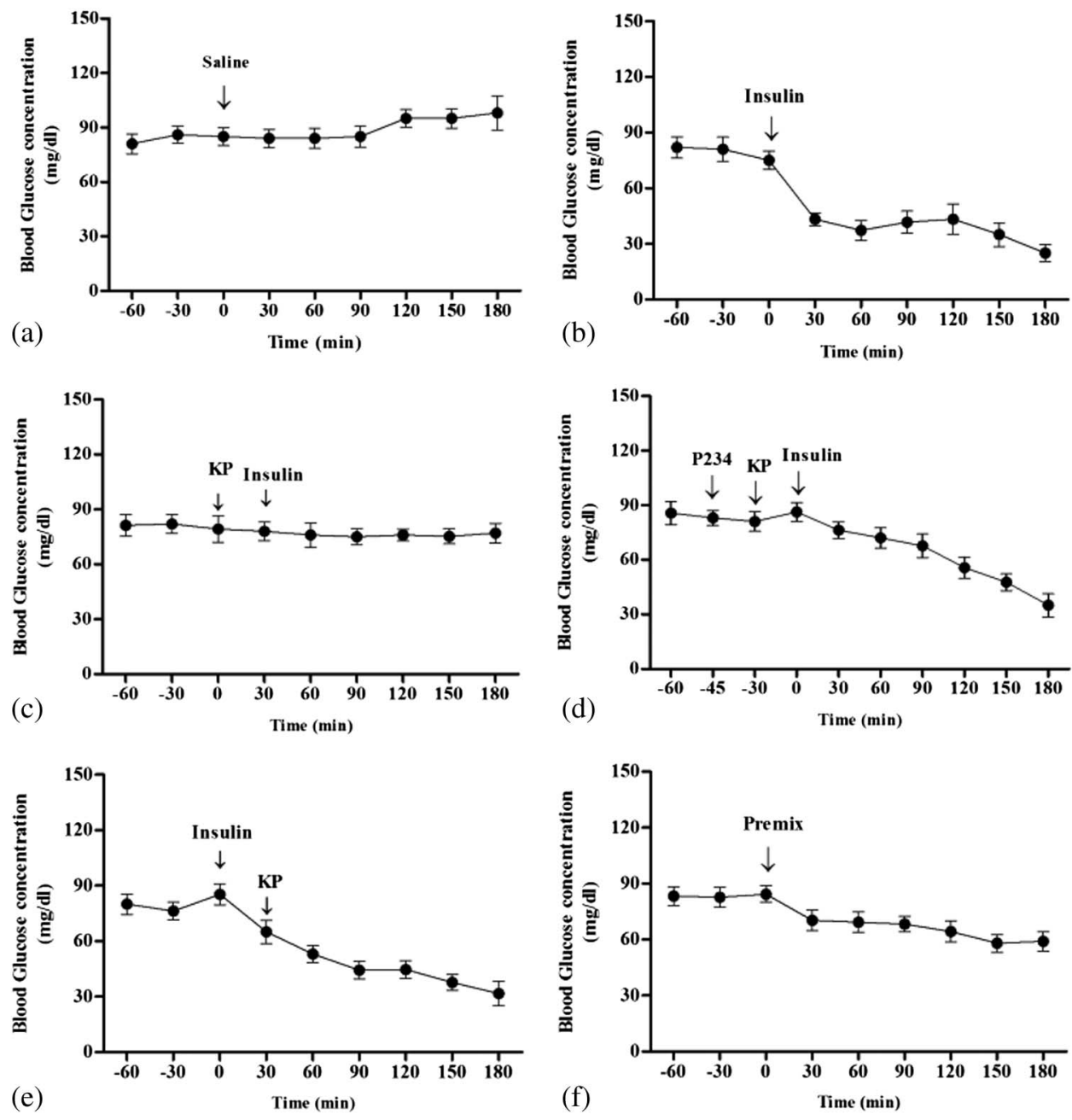

Fig. 4. Blood glucose levels $(\mathrm{mg} / \mathrm{dl})$ in healthy non-diabetic male rhesus monkeys at different time points for continuous $3 \mathrm{~h}$ (180 min): (a) saline treatment, (b) treatment with insulin alone, (c) KP-10 administration prior to insulin treatment, (d) administration of KP-10 antagonist P234 prior to KP-10 treatment, (e) KP-10 administration post-insulin treatment, and (f) treatment with a combined premix dose of KP-10 and insulin.

Values are mean $\pm \mathrm{SE}$ 
As expected, upon insulin administration, chair-restraint monkeys showed significant decrease in circulating blood glucose levels at all time points. Blood glucose declined from 75 ( $0 \mathrm{~min}$ ) to $43 \mathrm{mg} / \mathrm{dl}$ by $30 \mathrm{~min}$ and radically to $25 \mathrm{mg} / \mathrm{dl}$ by $180 \mathrm{~min}(66 \%$ decline). Thus, post-insulin treatment, glucose concentrations showed a significant difference $(p<0.001)$ by 180 min compared to predose glucose concentrations (Fig. 4b).

Treatment with KP-10 half an hour before insulin administration brought about nonsignificant alteration in blood glucose levels over 3-h time duration, with nearly similar glucose levels at 0 and $180 \mathrm{~min}$ ( $79 \mathrm{vs} .77 \mathrm{mg} / \mathrm{dl}$, respectively; a 3.0\% decline) (Fig. 4c).

When KP-10 action was blocked by pre-treatment with P234 given at $-45 \mathrm{~min}$, i.e., $15 \mathrm{~min}$ before KP-10 administration (KP-10 given at $-30 \mathrm{~min}$ ), in healthy animals, glucose levels remained statistically indifferent among $-45,-30$, and 0 min time points. Administration of insulin at $0 \mathrm{~min}$ led to a significant and gradual decline in glucose concentration ( 86 vs. $35 \mathrm{mg} / \mathrm{dl}$ ) noticeable by $180 \mathrm{~min}$, representing a $59 \%$ decline from 0 min (Fig. 4d).

When monkeys were treated first with insulin and, after half an hour, injected with KP-10, glucose levels decreased significantly $(p<0.001)$ by $180 \mathrm{~min}$. At $0 \mathrm{~min}$, glucose concentration was $85 \mathrm{mg} / \mathrm{dl}$, which decreased to $65 \mathrm{mg} / \mathrm{dl}$ by $30 \mathrm{~min}$, immediately after which KP-10 was administered. The glucose concentration continued to decline afterward, until it reached $32 \mathrm{mg} / \mathrm{dl}$ by $180 \mathrm{~min}$, corresponding to an overall $62 \%$ decline in glucose concentration at $180 \mathrm{~min}$ compared to $0 \mathrm{~min}$ (Fig. $4 \mathrm{e}$ ).

Administration of premix combined dose of KP-10 and insulin resulted in a statistically significant decrease $(p<0.001)$ in glucose levels up to $180 \mathrm{~min}$ (glucose levels: $84 \mathrm{vs.} 59 \mathrm{mg} / \mathrm{dl}$ at 0 and $180 \mathrm{~min}$, respectively; Fig. 4f).

\section{Intergroup comparison of healthy monkeys at $180 \mathrm{~min}$}

Mean blood glucose concentrations between groups revealed that healthy monkeys treated with KP-10 $30 \mathrm{~min}$ before insulin administration showed significantly higher $(p<0.001)$ blood glucose levels at $180 \mathrm{~min}$ as compared to animals treated with insulin alone. Similarly, animals treated with a premix dose of KP-10 and insulin also showed a significant difference $(p<0.01)$ from animals treated with insulin alone in the same time interval. Whereas animals treated with KP-10 after 30 min of insulin administration showed no significant difference from the group treated with insulin alone. Treatment with P234 KP-10 antagonist blocked the effect of KP-10, resulting in significantly decreased glucose levels following insulin

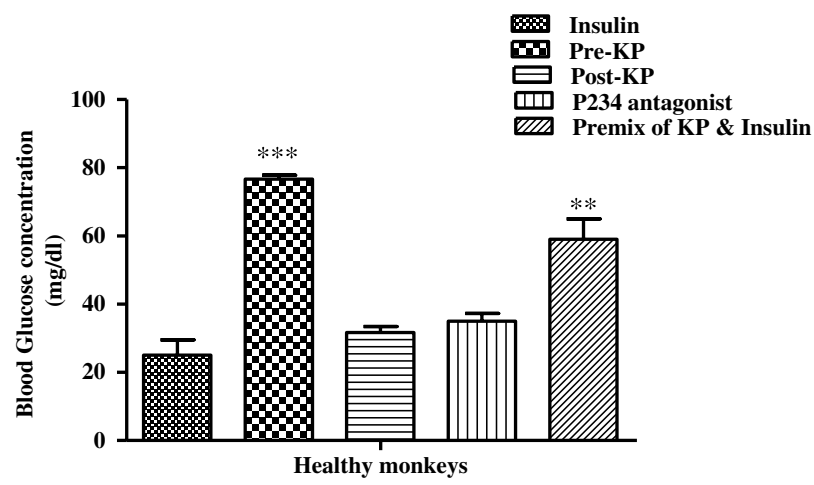

Fig. 5. Intergroup comparisons of blood glucose in healthy monkeys. Blood glucose levels $(\mathrm{mg} / \mathrm{dl})$ of experimental groups and the group treated with insulin alone at $180 \mathrm{~min}$ $(* * p<0.01 . * * * p<0.001)$. Values are mean $\pm \mathrm{SE}$ 
treatment, with glucose concentration at 180 min remaining comparable to that in the blood of the group treated with insulin alone (Fig. 5).

\section{Discussion}

Previous studies $(3,20,24)$ demonstrated the glucoregulatory role of kisspeptin, a reproduction-related peptide. Interaction of kisspeptin with other hormones such as insulin is also known (24). Hypoglycemic shock is a common occurrence associated with insulin therapy in diabetic patients (15). The question investigated in this study was whether kisspeptin prevents the animals from developing insulin-induced hypoglycemic shock. To this end, healthy and diabetic monkeys were subjected to kisspeptin and insulin treatments. The first phase of the study was crucial due to adequate selection of animal species and establishment of a diabetic animal model comparable to those of human beings. This involved rigorous chair restraining of rhesus monkeys to minimize the handling and experimental stress. Later, these monkeys were made diabetic with a single injection of STZ given i.v., followed by standard follow-up of the disease along with proper insulin treatment of each individual animal. To maintain hypoglycemic state, diabetic animals required comparatively higher doses of insulin, i.e., 7-11 IU/kg b.w. In addition, ketamine increases the blood glucose level in STZ-treated rats (5). This might be the reason why diabetic monkeys also needed higher insulin doses to cause a significant decrease in circulating glucose levels.

The results of this study showed that in the group where both healthy and diabetic monkeys were pre-treated with KP-10 followed by insulin treatment, circulating glucose levels did not decline to alarmingly low levels that were encountered during treatment with insulin alone. However, intragroup comparisons showed a significant decrease in blood glucose level in diabetic monkeys only. This differential response to KP-10 pre-treatment in healthy and diabetic animals was most likely due to the compromised glucoregulatory mechanisms of diabetic animals, which caused a significant decrease in blood glucose level by $180 \mathrm{~min}$ as compared to pre-treatment readings. However, KP-10 protected the circulating glucose levels from declining to levels approaching hypoglycemic shock, as was indicated by comparisons with treatments with insulin alone in the case of both healthy and diabetic animals.

Interestingly, the preventive action of kisspeptin against the effect of insulin on blood glucose was blocked when the kisspeptin antagonist P234 was administered prior to kisspeptin treatment. In alternate sets of experiments, when KP-10 and insulin were premixed and administered as a combined dose, this also prevented hypoglycemic shock. However, KP-10 infusion failed to prevent those monkeys from developing hypoglycemic shock who received insulin treatment prior to kisspeptin treatment.

Thus, the outcomes of our present in vivo study on rhesus monkeys confirm the glucoregulatory role of KP-10, corroborating earlier studies, which described the glucoregulatory role of kisspeptin. Silvestre et al. (19) reported the inhibitory effect of kisspeptin on beta cells obtained from perfused rat pancreas. Later, Bowe et al. (3) performed in vitro experiments on isolated mouse pancreatic islets and demonstrated that in the presence of stimulatory glucose levels, kisspeptin promotes insulin secretion. In addition, the study further demonstrated that administration of exogenous kisspeptin also exerted a stimulatory effect on insulin secretion but without changing the circulating glucose levels. This indicated an intact autoregulatory mechanism. 
Compared to an in vitro environment, the complex in vivo environment is influenced simultaneously by several nutritional, hormonal, and neuronal cues. Thus, exposure of pancreatic islets to various kisspeptin concentrations in vitro cannot be compared with in vivo conditions provided that the pharmacokinetics of kisspeptin is also considered (3). Therefore, the effects of KP-10 on circulating glucose concentration appear intricate when it comes to physiological interpretation. In the present work, kisspeptin was infused to monkeys at the dose of $50 \mu \mathrm{g}$, which was in fact a bolus dose within the supraphysiological range. It had to be kept at this high level, because the insulin action was extremely rapid and could easily lead to death of the monkeys. Moreover, this study aimed to evaluate the therapeutic potential of KP, about which the literature is still deficient. Current doses were optimized by initial trials, in which low to high $(10,100$, and $500 \mathrm{ng}$ as well as 1,10 , and $20 \mu \mathrm{g})$ kisspeptin doses failed to have any countering effect against insulin treatment. The study by Wahab et al. (24) also documented that peripheral administration of a bolus dose of KP-10 $(50 \mu \mathrm{g})$ did not change mean basal plasma insulin secretion in either fasted or fed monkeys, but appeared to be involved in the potentiation of glucose-induced insulin secretion. Furthermore, it was already known that the half life of KP-10 is approximately 4 min (8). This was also taken into consideration while optimizing kisspeptin doses, because a bolus dose could be expected to exert its glucoregulatory effect more effectively. As kisspeptin is an endogenous peptide and no toxic effects have been reported, investigations using its bolus dose as a potential drug are justified by previous studies $(6,17)$.

The results of previous in vitro studies $(10,16,19,20,23)$ have been contradictory as regards the stimulatory and inhibitory effect of kisspeptin on glucose-induced insulin secretion. These studies explained that this might have been due to the use of micromolar and nanomolar dose concentrations, respectively. Similarly, a cross-sectional observational study (1) carried out to investigate the association of kisspeptin levels with insulin secretion in non-diabetic humans concluded that kisspeptin concentrations in these individuals were significantly associated with decreases in glucose-stimulated insulin secretion.

In comparison, we focused on the experimentally induced diabetic state to investigate the glucoregulatory but preventive effect of KP-10 in vivo in diabetic monkeys, an effect that has not been reported earlier. Interestingly, our results revealed that KP-10, if administered prior to insulin challenge, bears the potential to prevent diabetic monkeys from hypoglycemic shock. A suppression of KP-10-induced glucoregulation by the kisspeptin antagonist P234 to separate groups of monkeys confirmed the preventive role of kisspeptin against insulininduced hypoglycemia and provided further strength to our hypothesis.

As to how kisspeptin could have achieved this goal is next question to answer. Possible explanations could be the mobilization of glucose from stores in the liver or stimulation of glucagon by the Kiss 1 peptide. Song et al. (20) found that hepatic kisspeptin levels increased in mice injected with glucagon $(16 \mu \mathrm{g} / \mathrm{kg})$ and that exposure of mouse hepatocytes to insulin $(2,000 \mathrm{pg} / \mathrm{ml})$ led to a reduction in Kiss 1 expression. However, it should be noted that physiological responses against insulin-induced hypoglycemia in experimental conditions may be different from those reported in human patients, which occur without any intervention and are usually caused by deficiency of any counter-regulatory hormone (18).

Administration of kisspeptin prior to insulin administration and the premix dose of kisspeptin and insulin, however, still indicates that kisspeptin, by an unknown mechanism, counteracted the hypoglycemic action of insulin. This countering effect of kisspeptin was abolished upon application of the P234 antagonist. It would be thus interesting to investigate 
the interaction of kisspeptin with the insulin receptor, and vice versa. Furthermore, treatment with kisspeptin when insulin had already been administered appears to be of no value, as it appears that insulin rapidly acted to lower glucose levels soon after its administration.

Although every effort was made to minimize stress due to handling or drugs intervention, some amount of stress could still have remained. This might have stimulated cortisol release. At present, however, the role of cortisol and growth hormone in stabilizing the circulating glucose level was not taken into consideration, because it was already known that cortisol does not play a significant role in the upregulation of glucose levels during acute insulin-induced hypoglycemia (7).

Current results obtained from both healthy and diabetic animals predict that the pharmacodynamics of exogenous kisspeptin overlaps with insulin, ultimately eliciting the appropriate physiological response to maintain relatively high blood glucose concentrations when it normally approaches to dangerously low levels.

Earlier experiments carried out in our laboratory on rats demonstrated a similar preventive action of KP-10 from hypoglycemic shock following insulin administration in alloxan-induced diabetic condition (data not shown). Our present results in monkeys further verify and indicate the efficacy of the Kiss-1 peptide in the maintenance of glucose homeostasis and to help prevent complications like sudden drops in blood glucose level, if used during insulin therapy for treatment of diabetes. The present and previous studies that have debated the stimulatory or inhibitory effect of kisspeptin on glucose-stimulated insulin secretion also encourage further investigation on its therapeutic efficacy in diabetes management.

\section{Conclusions}

This study attempted to determine the pharmacodynamics of peripherally administered KP-10 in the presence of insulin overload by directly measuring the circulating blood glucose concentration. To the best of the authors' knowledge, it is the first in vivo pharmacological study performed on rhesus monkeys that demonstrated the preventive effect of peripherally administered bolus dose of KP-10 against insulin-induced hypoglycemic shock in diabetic and healthy conditions.

\section{Acknowledgements}

The authors would like to thank Quaid-i-Azam University, Islamabad for funding the present research. No external funding was provided by any person or organization for this research.

\section{Conflict of interest}

The authors declare no conflict of interest.

\section{REFERENCES}

1. Andreozzi F, Mannino GC, Mancuso E, Spiga R, Perticone F, Sesti G: Plasma kisspeptin levels are associated with insulin secretion in nondiabetic individuals. PLoS One 12, 1-9 (2017)

2. Anjum S, Krishna A: Kisspeptin acts as a common messenger in the pathways controlling nutrition and reproduction in male mouse. Eur. J. Biomed. Pharm. Sci. 4, 317-329 (2017)

3. Bowe JE, King AJ, Kinsey-Jones JS, Foot VL, Li XF, O’Byrne KT, Persaud SJ, Jones PM: Kisspeptin stimulation of insulin secretion: mechanisms of action in mouse islets and rats. Diabetologia 52, 855-862 (2009) 
4. Budnitz DS, Lovegrove MC, Shehab N, Richards CL: Emergency hospitalizations for adverse drug events in older Americans. N. Engl. J. Med. 365, 2002-2012 (2011)

5. Chen Y, Li L, Xia H: Diabetes alters the blood glucose response to ketamine in streptozotocin-diabetic rats. Int. J. Clin. Exp. Med. 8, 11347-11351 (2015)

6. Ciaramella V, Corte CMD, Ciardiello F, Morgillo F: Kisspeptin and cancer: molecular interaction, biological functions, and future perspectives. Front. Endocrinol. 9, 115 (2018)

7. Cryer PE (1993): Glucose counterregulation: the physiological mechanisms that prevent or correct hypoglycaemia. In: Hypoglycaemia and Diabetes: Clinical and Physiological Aspects, eds Frier BM, Fisher BM, Edward Arnold, London, pp. 34-55

8. de Tassigny XA, Jayasena CN, Murphy KG, Dhillo WS, Colledge WH: Mechanistic insights into the more potent effect of KP-54 compared to KP-10 in vivo. PLoS One 12, e0176821 (2017)

9. Harwood H, Listrani P, Wagner J: Nonhuman primates and other animal models in diabetes research. J. Diabetes Sci. Technol. 6, 503-514 (2012)

10. Hauge-Evans AC, Richardson CC, Milne HM, Christie MR, Persaud SJ, Jones PM: A role for kisspeptin in islet function. Diabetologia 49, 2131-2135 (2006)

11. Huma T, Ulla F, Hanif F, Rizak JD, Shahab M: Peripheral administration of kisspeptin antagonist does not alter basal plasma testosterone but decreases plasma adiponectin levels in adult male rhesus macaques. Turk. J. Biol. $38,450-456$ (2014)

12. Hussain MA, Akalestou E, Song WJ: Inter-organ communication and regulation of beta cell function. Diabetoloogia 59, 659-667 (2016)

13. Hussain MA, Song WJ, Wolfe A: There is kisspeptin - and then there is kisspeptin. Trends Endocrinol. Metab. 26, 564-572 (2015)

14. Irwin N, Flatt PR: New perspectives on exploitation of incretin peptides for the treatment of diabetes and related disorders. World J. Diabetes 6, 1285-1295 (2015)

15. Ortiz MR: Hypoglycemia in diabetes. Nurs. Clin. North Am. 52, 565-574 (2017)

16. Schwetz TA, Reissaus CA, Piston DW: Differential stimulation of insulin secretion by GLP-1 and kisspeptin-10. PLoS One 9, e113020 (2014)

17. Seminara SB, Dipietro MJ, Ramaswamy S, Crowley WF Jr, Plant TM: Continuous human metastin 45-54 infusion desensitizes $\mathrm{G}$ protein-coupled receptor 54-induced gonadotropin-releasing hormone release monitored indirectly in the juvenile male rhesus monkey (Macaca mulatta): A finding with therapeutic implications. Endocrinology 147, 2122-2126 (2006)

18. Service FJ: Hypoglycemic disorders. N. Engl. J. Med. 332, 1144-1152 (1995)

19. Silvestre RA, Egido EM, Hernandez R, Marco J: Kisspeptin-13 inhibits insulin secretion without affecting glucagon or somatostatin release: study in the perfused rat pancreas. J. Endocrinol. 196, 283-290 (2008)

20. Song WJ, Mondal P, Wolfe A, Alonso LC, Stamateris R, Ong BWT, Lim OC, Yang KS, Radovick S, Novaria HJ, Farber EA, Farber CR, Turner SD, Hussain MA: Glucagon regulates hepatic kisspeptin to impair insulin secretion. Cell Metab. 19, 667-681 (2014)

21. Tesfaye N, Seaquist ER: Neuroendocrine responses to hypoglycemia. Ann. N. Y. Acad. Sci. 1212, 12-28 (2010)

22. Tritschler S, Theis FJ, Lickert H, Böttcher A: Systematic single-cell analysis provides new insights into heterogeneity and plasticity of the pancreas. Mol. Metab. 6, 974-990 (2017)

23. Vikman J, Ahren B: Inhibitory effect of kisspeptins on insulin secretion from isolated mouse islets. Diabetes Obes. Metab. 11, 197-201 (2009)

24. Wahab F, Riaz T, Shahab M: Study on the effect of peripheral kisspeptin administration on basal and glucoseinduced insulin secretion under fed and fasting conditions in the adult male rhesus monkey (Macaca mulatta). Horm. Metab. Res. 43, 37-42 (2011) 